Honam Mathematical J. 36 (2014), No. 3, pp. 505-517

http://dx.doi.org/10.5831/HMJ.2014.36.3.505

\title{
JORDAN HIGHER CENTRALIZERS ON SEMIPRIME RINGS AND RELATED MAPPINGS
}

\author{
YONG-SOO JUNG
}

\begin{abstract}
We prove that every Jordan higher left (right) centralizer on a 2-torsion free semiprime ring is a higher left (right) centralizer which is to generalize the result of Zalar [18].
\end{abstract}

\section{Introduction and Preliminaries}

Throughout this note, $R$ will represent an associative ring. For $a, b \in$ $R$, let $a b-b a$ be denoted by $[a, b]$ and let $\mathbb{N}$ be the set of all positive integers.

A derivation (resp. Jordan derivation) is an additive mapping $\delta$ : $R \rightarrow R$ satisfying $\delta(a b)=a \delta(b)+\delta(a) b$ for all $a, b \in R\left(\right.$ resp. $\delta\left(a^{2}\right)=$ $a \delta(a)+\delta(a) a$ for all $a \in R$ ). A left (resp. right) centralizer of $R$ is an additive mapping $\varphi: R \rightarrow R$ which satisfies $\varphi(a b)=\varphi(a) b$ (resp. $\varphi(a b)=a \varphi(b))$ for all $a, b \in R$. If $a, x \in R$, then $L_{x}(a)=x a$ is a left centralizer and $R_{x}(a)=a x$ is a right centralizer.

It is obvious that every derivation is a Jordan derivation. But the converse is in general not true. Herstein [10] proved that the converse is true on 2-torsion free prime rings and latter on, Brešar [5] extended this result to 2-torsion free semiprime rings. Brešar, Vukman ([6], [15]), Deng [8] and Ashraf et al. [1] studied Jordan left derivations and left derivations on prime rings and semiprime rings, which are in a close connection with so-called commuting mappings. In [18], Zalar proved that every Jordan left (right) centralizer on a 2-torsion free semiprime ring is a left (right) centralizer.

Received April 30, 2014. Accepted June 17, 2014.

2010 Mathematics Subject Classification. 16W25, 16N60, 16 U80.

Key words and phrases. semiprime ring, higher left (right) centralizer, Jordan higher left (right) centralizer, higher derivations, Jordan higher derivations, generalized higher derivations, generalized Jordan higher derivations. 
In [3], Brešar defined the following concept. An additive map $f$ : $R \rightarrow R$ is called a generalized derivation if there exists a derivation $\delta: R \rightarrow R$ such that $f(a b)=a \delta(b)+f(a) b$ for all $a, b \in R$. This notion is found in [13] and other properties of generalized derivations were given by Hvala [11] and Quadri et al. [14]. We call an additive mapping $f: R \rightarrow R$ a generalized Jordan derivation if there exists a Jordan derivation $\delta: R \rightarrow R$ such that $f\left(a^{2}\right)=a \delta(a)+f(a) a$ for all $a \in R$ (see [2] and [12]).

Jing and $\mathrm{Lu} \mathrm{[12]} \mathrm{proved} \mathrm{that} \mathrm{every} \mathrm{generalized} \mathrm{Jordan} \mathrm{derivation} \mathrm{on}$ a 2-torsion free prime ring is a generalized derivation. Furthermore, Vukman [16] proved that this result remains valid for semiprime rings.

Now, we extend the above mappings to (Jordan) higher derivations, generalized (Jordan) higher derivations and related mappings which have been studied in rings (mainly in commutative rings), but also in noncommutative rings.

Definition 1.1. Let $\Delta=\left(\delta_{n}\right)_{n \in \mathbb{N}}$ be a family of additive mappings on a ring $R . \Delta$ is said to be:

(i) a higher derivation on $R$ if for each $n \in \mathbb{N}$,

$$
\delta_{n}(a b)=\sum_{i+j=n} \delta_{i}(a) \delta_{j}(b) \text { for all } a, b \in R ;
$$

(ii) a Jordan higher derivation on $R$ if for each $n \in \mathbb{N}$,

$$
\delta_{n}\left(a^{2}\right)=\sum_{i+j=n} \delta_{i}(a) \delta_{j}(a) \text { for all } a \in R,
$$

where $\delta_{0}=i d_{R}$ on $R$.

Definition 1.2. Let $H=\left(h_{n}\right)_{n \in \mathbb{N}}$ be a family of additive mappings on a ring $R$ and let $\Phi=\left(\varphi_{n}\right)_{n \in \mathbb{N}}$ be a family of additive mappings on $R$. $\Phi$ is said to be:

(i) a higher left (right) centralizer on $R$ associated with $H$ if for each $n \in \mathbb{N}$,

$\varphi_{n}(a b)=\sum_{i+j=n} \varphi_{i}(a) h_{j}(b)\left(\varphi_{n}(a b)=\sum_{i+j=n} h_{i}(a) \varphi_{j}(b)\right)$ for all $a, b \in R$;

(ii) a Jordan higher left (right) centralizer on $R$ associated with $H$ if for each $n \in \mathbb{N}$,

$\varphi_{n}\left(a^{2}\right)=\sum_{i+j=n} \varphi_{i}(a) h_{j}(a) \quad\left(\varphi_{n}\left(a^{2}\right)=\sum_{i+j=n} h_{i}(a) \varphi_{j}(a)\right)$ for all $a \in R$, 
where $h_{0}=i d_{R}$ and $\varphi_{0}=0$ on $R$.

Definition 1.3. Let $F=\left(f_{n}\right)_{n \in \mathbb{N}}$ be a family of additive mappings on a ring $R$.

(i) if $\Delta=\left(\delta_{n}\right)_{n \in \mathbb{N}}$ is a higher derivation on $R$ and for each $n \in \mathbb{N}$,

$$
f_{n}(a b)=\sum_{i+j=n} f_{i}(a) \delta_{j}(b) \text { for all } a, b \in R,
$$

then $F$ is said to be a generalized higher derivation on $R$ associated with $\Delta$;

(ii) if $\Delta=\left(\delta_{n}\right)_{n \in \mathbb{N}}$ is a Jordan higher derivation on $R$ and for each $n \in \mathbb{N}$,

$$
f_{n}\left(a^{2}\right)=\sum_{i+j=n} f_{i}(a) \delta_{j}(a) \text { for all } a \in R,
$$

then $F$ is said to be a generalized Jordan higher derivation on $R$ associated with $\Delta$, where $f_{0}=i d_{R}$ on $R$.

Remark 1.4. In preceding definitions, when $n=1$, (Jordan) higher derivations, generalized (Jordan) higher derivations and (Jordan) higher left (right) centralizers reduce to ordinary cases.

Ferrero and Haetinger [9] extended the Brešar's results in [4] and [5] to Jordan higher derivations. Cortes and Haetinger [7] proved that if $R$ is a 2-torsion free ring which has a commutator right nonzero divisor, then every generalized Jordan higher derivation is a generalized higher derivation. Recently, Wei and Xiao obtained some results for generalized Jordan higher derivations on semiprime rings [17].

In this paper we investigate that, by showing that every Jordan higher left (right) centralizer on a 2-torsion free semiprime ring is a higher left (right) centralizer, the notions of generalized higher derivation and generalized Jordan higher derivation on a 2 -torsion free semiprime ring are equivalent to each other.

\section{Technical Lemmas and Main Results}

We precede the proof of our main theorem by a series of lemmas.

Lemma 2.1 ([4, Lemma 1.2]). Let $G_{1}, G_{2}, \cdots, G_{n}$ be additive groups and $R$ be a semiprime ring. Suppose that mappings $S: G_{1} \times G_{2} \times \cdots \times$ 
$G_{n} \rightarrow R$ and $T: G_{1} \times G_{2} \times \cdots \times G_{n} \rightarrow R$ are additive in each argument. If we have

$$
S\left(a_{1}, a_{2}, \cdots, a_{n}\right) x T\left(a_{1}, a_{2}, \cdots, a_{n}\right)=0
$$

for all $x \in R, a_{i} \in G_{i}, i=1,2, \cdots, n$, then we get

$$
S\left(a_{1}, a_{2}, \cdots, a_{n}\right) x T\left(b_{1}, b_{2}, \cdots, b_{n}\right)=0
$$

for all $x \in R, a_{i}, b_{i} \in G_{i}, i=1,2, \cdots, n$.

Lemma 2.2. Let $R$ be a 2-torsion free ring equipped with a Jordan higher left centralizer $\Phi=\left(\varphi_{n}\right)_{n \in \mathbb{N}}$ associated with a Jordan higher derivation $\Delta=\left(\delta_{n}\right)_{n \in \mathbb{N}}$. Then for all $a, b, c \in R$ and $n \in \mathbb{N}$, we have

(i) $\varphi_{n}(a b+b a)=\sum_{i+j=n}\left[\varphi_{i}(a) \delta_{j}(b)+\varphi_{i}(b) \delta_{j}(a)\right]$;

(ii) $\varphi_{n}(a b a)=\sum_{i+j+k=n} \varphi_{i}(a) \delta_{j}(b) \delta_{k}(a)$;

(iii) $\varphi_{n}(a b c+c b a)=\sum_{i+j+k=n}\left[\varphi_{i}(a) \delta_{j}(b) \delta_{k}(c)+\varphi_{i}(c) \delta_{j}(b) \delta_{k}(a)\right]$.

Proof. The arguments used in [10] carry over almost verbatim, but we will proceed the short proofs for the sake of completeness.

(i) Since $\varphi_{n}\left(a^{2}\right)=\sum_{i+j=n} \varphi_{i}(a) \delta_{j}(a)$, we replace $a$ by $a+b$ to linearize this. Then we obtain

$$
\varphi_{n}(a b+b a)=\sum_{i+j=n}\left[\varphi_{i}(a) \delta_{j}(b)+\varphi_{i}(b) \delta_{j}(a)\right]
$$

for all $a, b \in R$.

(ii) Let $W=\varphi_{n}(a(a b+b a)+(a b+b a) a)$. Using Lemma 2.2(i), we see that

$$
\begin{aligned}
W= & \sum_{i+j+k=n} \varphi_{i}(a) \delta_{j}(a) \delta_{k}(b)+2 \sum_{i+j+k=n} \varphi_{i}(a) \delta_{j}(b) \delta_{k}(a) \\
& +\sum_{i+j+k=n} \varphi_{i}(b) \delta_{j}(a) \delta_{k}(a .)
\end{aligned}
$$

On the other hand, we get

$$
\begin{aligned}
W & =\varphi_{n}\left(\left(a^{2} b+b a^{2}\right)+2 a b a\right) \\
& =\sum_{i+j+k=n} \varphi_{i}(a) \delta_{j}(a) \delta_{k}(b)+\sum_{i+j+k=n} \varphi_{i}(b) \delta_{j}(a) \delta_{k}(a)+2 \varphi_{n}(a b a) .
\end{aligned}
$$

Comparing two expressions for $W$, the result holds.

(iii) We linearize the result of (ii) by replacing $a$ by $a+c$ and so arrive at the expression. 
Let $\Phi=\left(\varphi_{n}\right)_{n \in \mathbb{N}}$ be a Jordan higher left centralizer on a ring $R$ associated with a Jordan higher derivation $\Delta=\left(\delta_{n}\right)_{n \in \mathbb{N}}$ and let

$$
P_{n}(a, b)=\varphi_{n}(a b)-\sum_{i+j=n} \varphi_{i}(a) \delta_{j}(b)
$$

for all $a, b \in R$ and $n \in \mathbb{N}$.

The following lemma remarks two elementary properties of the just defined relation. Whereas linearity in each argument (ii) is an immediate consequence of the definition, the equality (i) can be easily obtained from Lemma 2.2(i).

Lemma 2.3. Let $R$ be a 2-torsion free ring equipped with a Jordan higher left centralizer $\Phi=\left(\varphi_{n}\right)_{n \in \mathbb{N}}$ associated with a Jordan higher derivation $\Delta=\left(\delta_{n}\right)_{n \in \mathbb{N}}$. Then for all $a, b \in R$ and $n \in \mathbb{N}$, we have

(i) $P_{n}(a, b)+P_{n}(b, a)=0$;

(ii) $P_{n}(a, b)$ is additive in each argument.

Lemma 2.4. Let $R$ be a 2-torsion free semiprime ring equipped with a Jordan higher left centralizer $\Phi=\left(\varphi_{n}\right)_{n \in \mathbb{N}}$ associated with a Jordan higher derivation $\Delta=\left(\delta_{n}\right)_{n \in \mathbb{N}}$. If $n \in \mathbb{N}$ is such that $P_{m}(a, b)=0$ for all $a, b \in R$ and $m<n$, then we have

$$
P_{n}(a, b) x[a, b]=0 \text { for all } a, b, x \in R .
$$

Proof. Since $R$ is semiprime, it follows from [9, Theorem 1.2] that $\Delta=$ $\left(\delta_{n}\right)_{n \in \mathbb{N}}$ is a higher derivation on $R$. Hence it is easy to see that

$$
\delta_{n}(a b c)=\sum_{i+j+k=n} \delta_{i}(a) \delta_{j}(b) \delta_{k}(c) \text { for all } a, b, c \in R .
$$

Let $W=\varphi_{n}(a b x b a+b a x a b)$. We compute $W$ in two ways. On the one hand, we have by Lemma 2.2(ii)

$$
\begin{aligned}
W= & \sum_{i+j+k=n} \varphi_{i}(a) \delta_{j}(b x b) \delta_{k}(a)+\sum_{i+j+k=n} \varphi_{i}(b) \delta_{j}(a x a) \delta_{k}(b) \\
= & \sum_{i+j+k=n} \varphi_{i}(a)\left(\sum_{p+q+r=j} \delta_{p}(b) \delta_{q}(x) \delta_{r}(b)\right) \delta_{k}(a) \\
& +\sum_{i+j+k=n} \varphi_{i}(b)\left(\sum_{p+q+r=j} \delta_{p}(a) \delta_{q}(x) \delta_{r}(a)\right) \delta_{k}(b) \\
= & \sum_{i+p+q+r+k=n} \varphi_{i}(a) \delta_{p}(b) \delta_{q}(x) \delta_{r}(b) \delta_{k}(a) \\
& +\sum_{i+p+q+r+k=n} \varphi_{i}(b) \delta_{p}(a) \delta_{q}(x) \delta_{r}(a) \delta_{k}(b)
\end{aligned}
$$




$$
\begin{aligned}
= & \sum_{\substack{i+p+q+r+k=n \\
q+r+k \neq 0}} \varphi_{i}(a) \delta_{p}(b) \delta_{q}(x) \delta_{r}(b) \delta_{k}(a)+\sum_{i+p=n} \varphi_{i}(a) \delta_{p}(b) x b a \\
& +\sum_{\substack{i+p+q+r+k=n \\
q+r+k \neq 0}} \varphi_{i}(b) \delta_{p}(a) \delta_{q}(x) \delta_{r}(a) \delta_{k}(b)+\sum_{i+p=n} \varphi_{i}(b) \delta_{p}(a) x a b .
\end{aligned}
$$

On the other hand, using the assumption $P_{m}(a, b)=0$ for all $a, b$ and $m<n$, we get

$$
\begin{aligned}
& W=\varphi_{n}((a b) x(b a))+\varphi_{n}((b a) x(a b)) \\
& =\sum_{i+j+k=n} \varphi_{i}(a b) \delta_{j}(x) \delta_{k}(b a)+\sum_{i+j+k=n} \varphi_{i}(b a) \delta_{j}(x) \delta_{k}(a b) \\
& =\sum_{i+j+k=n} \varphi_{i}(a b) \delta_{j}(x)\left(\sum_{p+q=k} \delta_{p}(b) \delta_{q}(a)\right) \\
& +\sum_{i+j+k=n} \varphi_{i}(b a) \delta_{j}(x)\left(\sum_{p+q=k} \delta_{p}(a) \delta_{q}(b)\right) \\
& =\sum_{i+j+p+q=n} \varphi_{i}(a b) \delta_{j}(x) \delta_{p}(b) \delta_{q}(a)+\sum_{i+j+p+q=n} \varphi_{i}(b a) \delta_{j}(x) \delta_{p}(a) \delta_{q}(b) \\
& =\sum_{\substack{i+j+p+q=n \\
j+p+q \neq 0}} \varphi_{i}(a b) \delta_{j}(x) \delta_{p}(b) \delta_{q}(a)+\varphi_{n}(a b) x b a \\
& +\sum_{\substack{i+j+p+q=n \\
j+p+q \neq 0}} \varphi_{i}(b a) \delta_{j}(x) \delta_{p}(a) \delta_{q}(b)+\varphi_{n}(b a) x a b \\
& =\sum_{\substack{i+j+p+q=n \\
j+p+q \neq 0}}\left(\sum_{u+v=i} \varphi_{u}(a) \delta_{v}(b)\right) \delta_{j}(x) \delta_{p}(b) \delta_{q}(a)+\varphi_{n}(a b) x b a \\
& +\sum_{\substack{i+j+p+q=n \\
j+p+q \neq 0}}\left(\sum_{u+v=i} \varphi_{u}(b) \delta_{v}(a)\right) \delta_{j}(x) \delta_{p}(a) \delta_{q}(b)+\varphi_{n}(a b) x a b \\
& =\sum_{\substack{u+v+j+p+q=n \\
j+p+q \neq 0}} \varphi_{u}(a) \delta_{v}(b) \delta_{j}(x) \delta_{p}(b) \delta_{q}(a)+\varphi_{n}(a b) x b a \\
& +\sum_{\substack{u+v+j+p+q=n \\
j+p+q \neq 0}} \varphi_{u}(b) \delta_{v}(a) \delta_{j}(x) \delta_{p}(a) \delta_{q}(b)+\varphi_{n}(a b) x a b .
\end{aligned}
$$

Comparing the last terms of two such expressions obtained for $W$, we obtain

$$
P_{n}(a, b) x b a+P_{n}(b, a) x a b=0 \text { for all } a, b, x \in R .
$$


Since $P_{n}(b, a)=-P_{n}(a, b)$ by Lemma $2.3(\mathrm{i})$, we see that $P_{n}(a, b) x[a, b]=0$ for all $a, b, x \in R$.

This completes the proof of the lemma.

Theorem 2.5. Let $R$ be a 2-torsion free semiprime ring equipped with a Jordan higher left centralizer $\Phi=\left(\varphi_{n}\right)_{n \in \mathbb{N}}$ associated with a Jordan higher derivation $\Delta=\left(\delta_{n}\right)_{n \in \mathbb{N}}$. If $n \in \mathbb{N}$ is such that $P_{m}(a, b)=0$ for all $a, b \in R$ and $m<n$, then we have

(i) $P_{n}(a, b) x[r, s]=0$ for all $a, b, x, r, s \in R$;

(ii) $P_{n}(a, b) \in Z(R)$ for all $a, b \in R$, where $Z(R)$ is the center of $R$;

(iii) $P_{n}(a, b)[r, s]=0$ for all $a, b, r, s \in R$.

Proof. (i) From Lemma 2.1 and Lemma 2.4, we immediately get.

(ii) Since we have, in view of (i),

$$
\begin{aligned}
& {\left[P_{n}(a, b), c\right] x\left[P_{n}(a, b), c\right]} \\
& =\left(P_{n}(a, b) c-c P_{n}(a, b)\right) x\left[P_{n}(a, b), c\right] \\
& =P_{n}(a, b) c x\left[P_{n}(a, b), c\right]-c P_{n}(a, b) x\left[P_{n}(a, b), c\right]=0
\end{aligned}
$$

for all $a, b, c \in R$, it follows from the semiprimness of $R$ that

$$
\left[P_{n}(a, b), c\right]=0 \text { for all } a, b, c \in R
$$

for all $a, b, c \in R$. This implies that $P_{n}(a, b) \in Z(R)$.

(iii) By (i) and (ii), it is clear.

Now we are ready to prove our main result which is to generalize the result of Zalar [18].

Theorem 2.6. Let $R$ be a 2-torsion free semiprime ring. Then every Jordan higher left centralizer $\Phi=\left(\varphi_{n}\right)_{n \in \mathbb{N}}$ on $R$ associated with a Jordan higher derivation $\Delta=\left(\delta_{n}\right)_{n \in \mathbb{N}}$ is a higher left centralizer $\Phi$ associated with a higher derivation $\Delta$.

Proof. As in the proof of Lemma 2.4, we first see that $\Delta$ is a higher derivation on $R$. By induction, we now intend to prove that $P_{n}(a, b)=0$ for all $a, b \in R$ and $n \in \mathbb{N}$. If $n=1$, then it follows from [18, Proposition 1.4] that $P_{1}(a, b)=0$ for all $a, b \in R$. Thus, we assume that $P_{m}(a, b)=0$ for all $a, b \in R$ and $m<n$. Then, from Theorem 2.5(i), we see that

$$
P_{n}(a, b) x[r, s]=0 \text { for all } a, b, x, r, s \in R .
$$

We have two cases:

Case I: $[r, s]=0$ is fulfilled for all $r, s \in R$ in (1), i.e., $R$ is commutative. 
Let $W=\varphi_{n}(a b x b a)$ for all $a, b, x \in R$. On the one hand we have

$$
\begin{aligned}
W & =\varphi_{n}(a(b x a) b) \\
& =\sum_{i+j+k=n} \varphi_{i}(a) \delta_{j}(b x a) \delta_{k}(b) \\
& =\sum_{i+j+k=n} \varphi_{i}(a)\left(\sum_{l+s+t=j} \delta_{l}(b) \delta_{s}(x) \delta_{t}(a)\right) \delta_{k}(b) \\
& =\sum_{i+l+s+t+k=n} \varphi_{i}(a) \delta_{l}(b) \delta_{s}(x) \delta_{t}(a) \delta_{k}(b) \\
& =\sum_{i+l+s+t+k=n} \varphi_{i}(a) \delta_{l}(b) \delta_{s}(x) \delta_{t}(a) \delta_{k}(b)+\sum_{i+l=n} \varphi_{i}(a) \delta_{l}(b) x a b .
\end{aligned}
$$

On the other hand we obtain, by using inductive hypothesis,

$$
\begin{aligned}
W & =\varphi_{n}((a b) x(a b)) \\
& =\sum_{i+j+k=n} \varphi_{i}(a b) \delta_{j}(x) \delta_{k}(a b) \\
& =\sum_{i+j+k=n} \varphi_{i}(a b) \delta_{j}(x)\left(\sum_{u+v=k} \delta_{u}(a) \delta_{v}(b)\right) \\
& =\sum_{i+j+u+v=n} \varphi_{i}(a b) \delta_{j}(x) \delta_{u}(a) \delta_{v}(b) \\
& =\sum_{\substack{i+j+u+v=n \\
j+u+v \neq 0}} \varphi_{i}(a b) \delta_{j}(x) \delta_{u}(a) \delta_{v}(b)+f_{n}(a b) x a b \\
& =\sum_{\substack{i+j+u+v=n \\
j+u+v \neq 0}}\left(\sum_{p+q=i} \varphi_{p}(a) \delta_{q}(b)\right) \delta_{j}(x) \delta_{u}(a) \delta_{v}(b)+\varphi_{n}(a b) x a b \\
& =\sum_{\substack{p+q+j+u+v=n \\
j+u+v \neq 0}} \varphi_{p}(a) \delta_{q}(b) \delta_{j}(x) \delta_{u}(a) \delta_{v}(b)+\varphi_{n}(a b) x a b .
\end{aligned}
$$

Comparing the last terms of two expressions for $W$, we have

$$
\left(\varphi_{n}(a b)-\sum_{i+l=n} \varphi_{i}(a) \delta_{l}(b)\right) x a b=0,
$$

that is,

$$
P_{n}(a, b) x a b=0 \text { for all } a, b, x \in R .
$$

Furthermore, the linearization of (2) is

$$
P_{n}(a, b) x y b=-P_{n}(y, b) x a b,
$$


and so (2) yields

$$
\left(P_{n}(a, b) x y b\right) z\left(P_{n}(a, b) x y b\right)=-P_{n}(a, b) x\left(y b z P_{n}(y, b)\right) x a b=0
$$

for all $a, b, x, y, z \in R$. Hence we obtain

$$
P_{n}(a, b) x y b=0 \text { for all } a, b, x, y \in R .
$$

Linearizing (3) and using the same approach as just done, we also have

$$
P_{n}(a, b) x y w=0 \text { for all } a, b, x, y, w \in R .
$$

In particular, we see that

$$
P_{n}(a, b) x y P_{n}(a, b) x=0 \text { for all } a, b, x, y \in R .
$$

Therefore, we get

$$
P_{n}(a, b) x=0 \text { for all } a, b, x \in R .
$$

From the semiprimeness of $R$, we conclude that

$$
P_{n}(a, b)=0 \text { for all } a, b \in R \text {. }
$$

Case II: There exist $r_{0}, s_{0} \in R$ satisfying $\left[r_{0}, s_{0}\right] \neq 0$ in (1).

From Theorem 2.5(iii), it follows that $P_{n}(a, b)\left[r_{0}, s_{0}\right]=0$ for all $a, b \in$ $R$. We claim that for any $t \in \mathbb{N}$ and for arbitrarily chosen $a, b \in R$, $\operatorname{Ann}\left(P_{n}(a, b)^{t}\right)$ is a semiprime ideal of $R$. In fact, from Theorem 2.5(ii), $\operatorname{Ann}\left(P_{n}(a, b)^{t}\right)$ is an ideal of $R$. For a given $x \in R$, suppose that $x r x \in$ $\operatorname{Ann}\left(P_{n}(a, b)^{t}\right)$ for all $r \in R$. Then we have $(x r x) P_{n}(a, b)^{t}=0$ which gives $\left(x P_{n}(a, b)^{t}\right) r\left(x P_{n}(a, b)^{t}\right)=0$. Hence the semiprimness of $R$ yields that $x \in \operatorname{Ann}\left(P_{n}(a, b)^{t}\right)$.

It is obvious that

$$
\operatorname{Ann}\left(P_{n}(a, b)^{t}\right) \subseteq \operatorname{Ann}\left(P_{n}(a, b)^{t+1}\right)
$$

for any $t \in \mathbb{N}$. It is easy to check that $I=\bigcup_{t \in \mathbb{N}} \operatorname{Ann}\left(P_{n}(a, b)^{t}\right)$ is also a semiprime ideal of $R$ and $R / I$ is a 2 -torsion free commutative semiprime ring by Theorem 2.5(iii). Now we assert that each additive mappings in $\Phi=\left(\varphi_{n}\right)_{n \in \mathbb{N}}$ and each additive mappings in $\Delta=\left(\delta_{n}\right)_{n \in \mathbb{N}}$ keep $I$ invariant, respectively. To get this assertion, we still use the induction. Let $a, b \in R$ be arbitrary and $s \in I$. If $n=1$, then there exists $t \in \mathbb{N}$ such that $s \in \operatorname{Ann}\left(P_{n}(a, b)^{t}\right)$. Therefore, we have

$$
0=\varphi_{1}\left(s P_{n}(a, b)^{t}\right)=\varphi_{1}(s) P_{n}(a, b)^{t},
$$

whence $\varphi_{1}(s) \in I$. This shows that $\varphi_{1}$ keeps $I$ invariant. As for $\delta_{1}$, we get

$$
0=\delta_{1}\left(s P_{n}(a, b)^{t}\right)=s \delta_{1}\left(P_{n}(a, b)^{t}\right)+\delta_{1}(s) P_{n}(a, b)^{t} .
$$


Right multiplication of (4) by $P_{n}(a, b)^{t}$ leads to

$$
\delta_{1}(s) P_{n}(a, b)^{2 t}=0 .
$$

Hence it follows that $\delta_{1}(s) \in I$ and $\delta_{1}$ keeps $I$ invariant. We assume that $\varphi_{m}(m<l)$ and $\delta_{m}(m<l)$ keep $I$ invariant. Then we have

$$
0=\varphi_{l}\left(s P_{n}(a, b)^{t}\right)=\varphi_{l}(s) P_{n}(a, b)^{t}+\sum_{\substack{i+j=l \\ i, j<l}} \varphi_{i}(s) \delta_{j}\left(P_{n}(a, b)^{t}\right) .
$$

According to the assumption, $\delta_{i}(s) \in I$ for all $i<l$. Right multiplication of (5) by $P_{n}(a, b)^{t}$ gives

$$
\varphi_{l}(s) P_{n}(a, b)^{2 t}=0 .
$$

This tells us that $\varphi_{l}$ keeps $I$ invariant.

As for $\delta_{l}, \delta_{0}=i d_{R}$ keeps $I$ invariant and we get

$$
\begin{aligned}
0 & =\delta_{l}\left(s P_{n}(a, b)^{t}\right) \\
& =s \delta_{l}\left(P_{n}(a, b)^{t}\right)+\delta_{l}(s) P_{n}(a, b)^{t}+\sum_{\substack{i+j=l \\
i, j<l}} \delta_{i}(s) \delta_{j}\left(P_{n}(a, b)^{t}\right) .
\end{aligned}
$$

In view of the assumptions, $\delta_{i}(s) \in I$ for all $i<l$. Right multiplication of (6) by $P_{n}(a, b)^{t}$ gives

$$
\delta_{l}(s) P_{n}(a, b)^{2 t}=0 .
$$

Thus $\delta_{l}(s) \in I$ and $\delta_{l}$ keeps $I$ invariant.

Hence each additive mappings in $\Phi$ and each additive mappings in $\Delta$ can be induced to a semiprime ring $R / I$ as follows:

$$
\tilde{\varphi}_{n}(\tilde{a})=\varphi_{n}(a)+I \text { and } \tilde{\delta}_{n}(\tilde{a})=\delta_{n}(a)+I
$$

for $\tilde{a} \in R / I$. It is easy to see that $\tilde{\Phi}=\left(\tilde{\varphi}_{n}\right)_{n \in \mathbb{N}}$ is a Jordan higher left centralizer on $R / I$ associated with a Jordan higher derivation $\tilde{\Delta}=$ $\left(\tilde{\delta}_{n}\right)_{n \in \mathbb{N}}$. Since $R / I$ is 2 -torsion free and commutative, it follows from Case I that $\tilde{P}_{n}(\tilde{a}, \tilde{b})=0$ which means that $P_{n}(a, b) \in I$. In particular, there exists $\nu \in \mathbb{N}$ such that $P_{n}(a, b) \in \operatorname{Ann}\left(P_{n}(a, b)^{\nu}\right)$. Therefore $P_{n}(a, b)^{\nu+1}=0$. However, there is no nonzero nilpotent element in the center of a semiprime ring. Since $a, b$ were arbitrarily chosen in $R$, we conclude that

$$
P_{n}(a, b)=0 \text { for all } a, b \in R .
$$

Thus $\Phi$ is a higher left centralizer on $R$ associated with a higher derivation $\Delta$. This completes the proof of the theorem. 
Remark 2.7. Since the concept of a Jordan higher left and right centralizer is symmetric, the conclusion is also valid for the right case of the preceding results.

The following is a result concerning the structure of generalized Jordan higher derivations.

Theorem 2.8. Let $R$ be a 2-torsion free ring and let $\Delta$ be a Jordan higher derivation on $R$. Then $F=\left(f_{n}\right)_{n \in \mathbb{N}}$ is a generalized Jordan higher derivation on $R$ associated with $\Delta=\left(\delta_{n}\right)_{n \in \mathbb{N}}$ if and only if there exists a Jordan higher left centralizer $\Phi=\left(\varphi_{n}\right)_{n \in \mathbb{N}}$ on $R$ associated with $\Delta$ such that $f_{n}=\varphi_{n}+\delta_{n}$ for all $n \in \mathbb{N}$.

Proof. Suppose that $F$ is a generalized higher derivation on $R$ associated with $\Delta$. Then

$$
f_{n}\left(a^{2}\right)=\sum_{i+j=n} f_{i}(a) \delta_{j}(a)
$$

holds for all $a, b \in R$. Let $\varphi_{n}=f_{n}-\delta_{n}$ for all $n \in \mathbb{N}$. Then, for each $n \in \mathbb{N}, \varphi_{n}$ is a linear mapping on $R$ and we have

$$
\begin{aligned}
\varphi_{n}\left(a^{2}\right) & =f_{n}\left(a^{2}\right)-\delta_{n}\left(a^{2}\right) \\
& =\sum_{i+j=n} f_{i}(a) \delta_{j}(a)-\sum_{i+j=n} \delta_{i}(a) \delta_{j}(a) \\
& =\sum_{i+j=n}\left[f_{i}(a)-\delta_{i}(a)\right] \delta_{j}(a)=\sum_{i+j=n} \varphi_{i}(a) \delta_{j}(a)
\end{aligned}
$$

for all $a \in A$. Therefore $\Phi$ is a Jordan higher left centralizer on $R$ associated with $\Delta$ and $f_{n}=\varphi_{n}+\delta_{n}$ for all $n \in \mathbb{N}$.

Conversely, let $\Phi$ be a Jordan higher left centralizer on $R$ associated with $\Delta$ such that $f_{n}=\varphi_{n}+\delta_{n}$ for all $n \in \mathbb{N}$. Then, for each $n \in \mathbb{N}, f_{n}$ is an additive mapping on $R$ and we get

$$
\begin{aligned}
f_{n}\left(a^{2}\right) & =\varphi_{n}\left(a^{2}\right)+\delta_{n}\left(a^{2}\right) \\
& =\sum_{i+j=n} \varphi_{i}(a) \delta_{j}(a)+\sum_{i+j=n} \delta_{i}(a) \delta_{j}(a) \\
& =\sum_{i+j=n}\left[\varphi_{i}(a)+\delta_{i}(a)\right] \delta_{j}(a)=\sum_{i+j=n} f_{i}(a) \delta_{j}(a)
\end{aligned}
$$

for all $a \in A$. Thus $F$ is a generalized Jordan higher derivation on $R$ associated with $\Delta$.

Using Theorem 2.6 and Theorem 2.8, we have our final result: 
Corollary 2.9. Let $R$ be a 2-torsion free semiprime ring. Then every generalized Jordan higher derivation $F=\left(f_{n}\right)_{n \in \mathbb{N}}$ on $R$ associated with a Jordan higher derivation $\Delta=\left(\delta_{n}\right)_{n \in \mathbb{N}}$ is a generalized higher derivation on $R$ associated with a higher derivation $\Delta$.

Proof. From Theorem 2.8, it follows that there exists a Jordan higher left centralizer $\Phi=\left(\varphi_{n}\right)_{n \in \mathbb{N}}$ on $R$ associated with $\Delta$ such that $f_{n}=\varphi_{n}+\delta_{n}$ for all $n \in \mathbb{N}$.

Since $R$ is semiprime, we see that $\Delta$ is a higher derivation in view of $[9$, Theorem 1.2] and $\Phi$ is a higher left centralizer associated with $\Delta$ by Theorem 2.6. This means that $F$ is a generalized higher derivation on $R$ associated with $\Delta$. The proof of the theorem completes.

\section{References}

[1] M. Ashraf, N. Rehman and S. Ali, On Jordan left derivations of Lie ideals in prime rings, Southeast Asian Bull. Math. 25(3) (2001), 379-382.

[2] M. Ashraf and N. Rehman and Shakir Ali, On Lie ideals and Jordan generalized derivations of prime rings, Indian J. pure appl. Math. 34(2) (2003), 291-294.

[3] M. Brešar, On the distance of the compositions of two derivations to the generalized derivations, Glasgow Math. J. 33 (1991), 89-93.

[4] _ Jordan mappings of semiprime rings, J. Algebra, 127 (1989), 218-228.

[5] _ Jordan derivations on semiprime rings, Proc. Amer. Math. Soc. 104 (1988), 1003-1006.

[6] M. Brešar and J. Vukman, On left derivations and related mappings, Proc. Amer. Math. Soc. 10 (1990), 7-16.

[7] W. Cortes and C. Haetinger, On Jordan generalized higher derivations in rings, Turkish J. Math. 29 (2005), 1-10.

[8] Q. Deng, On Jordan left derivations, Math. J. Okayama Univ. 34 (1997), 145147.

[9] M. Ferrero and C. Haetinger, Higher derivations and a theorem by Herstein, Quaestiones Mathematicae 25(2) (2002), 249-257.

[10] I. N. Herstein, Jordan derivations of prime rings, Proc. Amer. Math. Soc. 8 (1957), 1104-1110.

[11] B. Hvala, Generalized derivations in rings, Comm. Algebra 26(4) (1998), 11471166.

[12] W. Jing and S. Lu, Generalized Jordan derivations on prime rings and standard operator algebras, Taiwanese J. Math. 7(4) (2003), 605-613.

[13] P. Ribenboim, Higher order derivations of modules, Portgaliae Math. 39 (1980), 381-397.

[14] M. A. Quadri, M. Shadab Khan and N. Rehman, Generalized derivations and commutativity of prime rings, Indian J. Pure Appl. Math. 34(9) (2003), 13931396.

[15] J. Vukman, Jordan left derivations on semiprime rings, Math. J. Okayama Univ. 39 (1997), 1-6. 
[16] _ A note on generalized derivations of semiprime rings, Taiwanese J. Math. 11(2) (2007), 367-370.

[17] F. Wei and Z. Xiao, Generalized Jordan triple higher derivations on rings, B. Korean Math. Soc. 46(3) (2009), 553-565 .

[18] B. Zalar, On centralizers of semiprime rings, Comment. Math. Univ. Carolinae 32(4) (1991), 609-614.

Yong-Soo Jung

Department of Mathematics, Sun Moon University, Asan, Chungnam 336-708, Korea.

E-mail: ysjung@sunmoon.ac.kr 